'Sección Reumatología, Hospital Clínico Universidad de Chile. 25ección Medicina Interna, Hospital Clínico Universidad de Chile.

${ }^{3}$ Facultad de Medicina Universidad de Concepción, Chile.

${ }^{4}$ Hospital Las Higueras de Talcahuano, Chile.

${ }^{5}$ Servició de Imagenología Hospital Clínico Universidad de Chile.

'Servicio de Anatomía Patológica, Hospital Clínico Universidad de Chile.

anterno de Medicina, Hospital Clínico Universidad de Chile.

Sin Financiamiento.

Recibido el 7 de noviembre de 2013, aceptado el 12 de mayo de 2014.

Correspondencia a: Pamela Wurmann Kiblisky Teléfono: 56-2-29788236 pwurmann@hcuch.ce

\section{Aortitis, causas infrecuentes en Reumatología: presentación de casos}

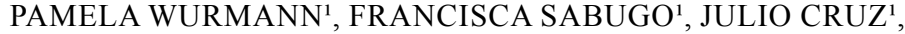 \\ GONZALO DÍAZ ${ }^{2}$, FELIPE SÁNCHEZ ${ }^{\mathrm{a}}$, SANDRA PINO ${ }^{3}$, NINETTE PEZO ${ }^{4}$, \\ JUAN CARLOS DÍAZ ${ }^{5}$, CRISTINA FERNÁNDEZ ${ }^{6}$
}

\section{Aortitis. Report of three cases}

Aortitis is a nonspecific term that describes an inflammation of the aortic wall caused by inflammatory, infectious, paraneoplastic and idiopathic diseases. The symptoms are variable and nonspecific; therefore a high level of clinical suspicion is required to diagnose it. It is often an incidental finding while looking for other diagnoses and it is confirmed mainly through imaging studies. We report three cases of aortitis: A 29-year-old woman presenting with alopecia, oral and nasal ulcers and positive antinuclear antibodies. A CAT scan showed a segmental thickening of thoracic aorta, with dilated and stenotic areas. She was successfully treated with steroids, hydroxychloroquine, cyclophosphamide and azathioprine. A 41-year-old male presenting with dorsal pain and cough. The CAT scan showed an extra-intimal thickening of the descending aorta and stenosis of the celiac artery. The final diagnosis was a polyangiitis and was treated with steroids, cyclophosphamide and azathioprine. A 28-year-old woman presenting with pain in the left upper abdomen. Imaging studies showed a thickening of the aortic arch and subclavian artery. The final diagnosis was sarcoidosis and the patient was treated with prednisone.

(Rev Med Chile 2014; 142: 924-929)

Key words: Anti-Neutrophil Cytoplasmic Antibody-associated vasculitis; Aortitis; Lupus erythematosus systemic; Sarcoidosis.

\section{A} ortitis es un término inespecífico para describir inflamación de las paredes de la aorta. Existen diversas causas como enfermedades inflamatorias, infecciosas, paraneoplásicas y la idiopática ${ }^{1,2}$. Las causas infecciosas son raras, existiendo por bacterias (Salmonella, Staphylococcus o sifilítica) y micobacterias ${ }^{2}$. En las asociadas a neoplasia se han descrito casos de síndromes mielodisplásicos, entre otros ${ }^{3}$.

Dentro de las etiologías inflamatorias están las vasculitis de grandes vasos como la arteritis de células gigantes (ACG) y enfermedad de Takayasu (ET), el síndrome IgG4, policondritis recurrente, síndrome de Cogan. Se describe en frecuencia muy inferior en patologías como espondiloartropatías, Behçet, artritis reumatoide, lupus (LES) y casos aislados en sarcoidosis, granulomatosis con poliangeitis (PG, antiguo Wegener), poliarteritis nodosa ${ }^{4}$ y en el síndrome antifosfolípidos ${ }^{5}$.

La inflamación puede afectar los distintos segmentos aórticos lo que determinará en parte la sintomatología. La presentación clínica es inespecífica. Se confirma con angiotomografía computarizada (aTAC) o angioresonancia magnética (aRNM). La biopsia aporta poco en términos de establecer la causa subyacente y el tratamiento se orienta según la etiología.

A continuación se presentan casos de aortitis como manifestación infrecuente de 3 distintas enfermedades reumatológicas.

\section{Pacientes y Método}

Previa firma de consentimiento informado, se revisaron las historias clínicas de 3 pacientes con 
Aortitis en reumatología, causas infrecuentes - P. Wurmann et al

diagnóstico de aortitis asociada a otras enfermedades reumatológicas; se analizaron las imágenes por un radiólogo especializado.

\section{Caso 1}

Mujer de 29 años. Con historia de 3 años de diagnóstico de mesenquimopatía indiferenciada por nariz en silla de montar, sin historia de condritis. Anticuerpos antinucleares (ANA) y anticuerpos anti-Ro + . Consultó por alopecia, artritis, úlceras orales y nasales. Presentaba soplo aórtico sistólico V/VI irradiado al cuello. Tenía velocidad de eritrosedimentación (VHS) $64 \mathrm{~mm} / \mathrm{h}$, proteína C reactiva $(\mathrm{PCR}) 5(\mathrm{VN}<10 \mathrm{mg} / \mathrm{L})$, creatininemia $0,5 \mathrm{mg} / \mathrm{dl}$, ANA $1 / 160$ moteado, anti-Ro $22,5 \mathrm{U}$ ( VN < 15), anti-B2 glicoproteína 1 IgM 31,2 MPL $(\mathrm{VN}<8)$ e IgG 17 GPL $(\mathrm{VN}<8)$, examen de orina normal. Ecocardiograma: aceleración del flujo de aorta torácica y vasos del cuello. aTAC de tórax, cuello y cerebral: engrosamiento segmentario y realce con contraste de la pared aórtica y sus ramas principales, con áreas de estenosis y dilatación alternadas. Estenosis moderada de carótida derecha, origen del tronco celíaco y arteria mesentérica superior (Figuras 1A, 1B). Vasos cerebrales normales. Se trató con corticoides, hidroxicloroquina y 6 pulsos de ciclofosfamida, luego azatioprina y prednisona. El control a 6 meses demostró disminución de la inflamación de aorta abdominal, tronco celíaco y arteria mesentérica superior, estenosis de $50 \%$ del origen del tronco celíaco con dilatación post estenótica (Figuras 1C, 1D) y normalización de los parámetros inflamatorios. A 2 años de seguimiento la paciente se encuentra asintomática y con exámenes normales.

\section{Caso 2}

Hombre de 41 años, fumador. Historia de 2 meses de dolor dorsal irradiado a hombro izquierdo, intensidad 8/10 sin respuesta a analgésicos, tos con expectoración mucosa, baja de peso y sudoración nocturna. Examen físico inicial normal. Presentó anemia leve, VHS: $96 \mathrm{~mm} / \mathrm{h}$, examen de orina, función renal, pruebas hepáticas y perfil lipídico normales. Tomografía computada de columna dorsal sin hallazgos significativos. Radiografía de tórax: nódulos subpleurales en vértices pulmonares y lóbulo superior izquierdo. Hemocultivos negativos. Ecotomografía abdominal normal, aTAC de tórax ateromatosis aórtica segmentaria en aorta descendente, con engro-
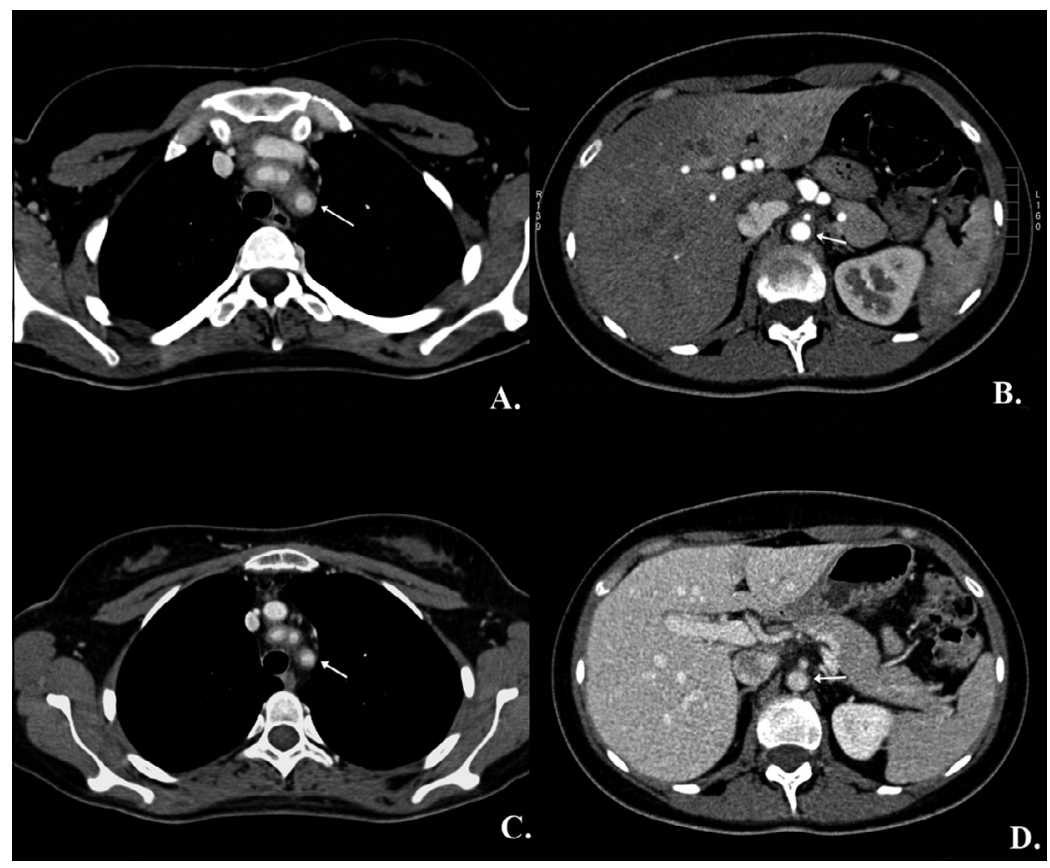

Figura 1. Cortes axiales de tomografía computada (TC) contrastada. Fila superior, al momento del diagnóstico. Se observa engrosamiento concéntrico y realce parietal de los troncos arteriales supra-aórticos (A), aorta abdominal proximal y emergencia de la arteria mesentérica superior (B). En la fila inferior, post tratamiento esteroidal, se objetiva una disminución del compromiso de dichas ramas arteriales (C, D). 
samiento extraintimal, estenosis significativa de ostium en tronco celíaco (Figuras 2a, 2b, 2c, 2d). Nódulos pulmonares y condensaciones apicales bilaterales. VDRL: No reactivo, VIH negativo, PPD: $10 \mathrm{~mm}$. Quatiferon (-). El paciente evolucionó con escleritis bilateral y fiebre hasta $37,8^{\circ} \mathrm{C}$. Se sospecha vasculitis de grandes vasos. Nuevos estudios demostraron ANCA citoplasmático $(C)$ : positivo, anti proteinasa $3(\mathrm{PR} 3)+6,5(\mathrm{VN}<1,2)$, C3 y C4 normales, ANA, anticuerpos anti-ADN y ENA negativos. Biopsia pulmonar: Nódulo con centro fibroso, necrosis y reacción histiocitaria, con células gigantes. Se reconoce neumonía organizativa periférica, signos de hemorragia antigua, concordante con PG. Recibió corticoterapia y ciclofosfamida oral por un año y posteriormente mantención con azatioprina. Presentó buena respuesta clínica, normalización de los parámetros inflamatorios, radiografía y aTAC de tórax. Anti PR3 (-) a los 6 meses.

\section{Caso 3}

Mujer de 28 años con antecedentes de hipotiroidismo y depresión en tratamiento. Presentó 8 días de dolor en flanco izquierdo y epigastrio, irradiado a zona lumbar, intensidad 7/10 que cedió parcialmente con analgesia, fatigabilidad, sensación febril no cuantificada, cefalea frontal y sudoración nocturna. Ingresó febril e hipotensa, dolor a la palpación profunda en flanco izquierdo y epigastrio, y disminución leve de pulso braquial y radial derecho. Destacó anemia leve, leucocitosis y trombocitosis leve, VHS: 89 y PCR: 264,4 $\mathrm{mg} / \mathrm{l}(\mathrm{VN}<10 \mathrm{mg} / \mathrm{l})$ función renal, sedimento urinario y perfil hepático normales. TAC de abdomen y pelvis: finas bandas atelectásicas basales y derrame pleural laminar izquierdo. Alteración de perfusión en riñón izquierdo sospechoso de pielonefritis aguda. Se hospitalizó con diagnóstico de sepsis de foco urinario y se inició tratamiento antibiótico. Hemocultivos y urocultivo fueron negativos. Cinética del fierro compatible con anemia de enfermedades crónicas. Persistió con dolor abdominal, parámetros inflamatorios elevados y fiebre hasta $38^{\circ} \mathrm{C}$. aTAC de tórax y cuello: adenopatías mediastínicas, paratraqueales, subcarinales e hiliares bilaterales simétricas. Aorta torácica de calibre normal, con engrosamiento parietal en arco y segmento descendente distal de aspecto inflamatorio, engrosamiento parietal de arteria subclavia izquierda (Figuras 3A, 3B). Se sospechó ET versus sarcoidosis.

ANA (+) 1/80 moteado, ENA (-), anti-ADN (-), C3 y C4 (normal), FR (-), Coombs directo (-), ANCA por IFI y Elisa (-), VDRL (-), VIH (-), Elispot (-).

Biopsia de adenopatías: compatible con sarcoidosis (Figuras 4a, 4b, 4c). Se indicó prednisona en dosis decrecientes hasta suspender a los 8 meses. $\mathrm{Al}$ año de seguimiento la paciente está asintomática y con aTAC (Figuras 3C, 3D) y todos los exámenes normales.

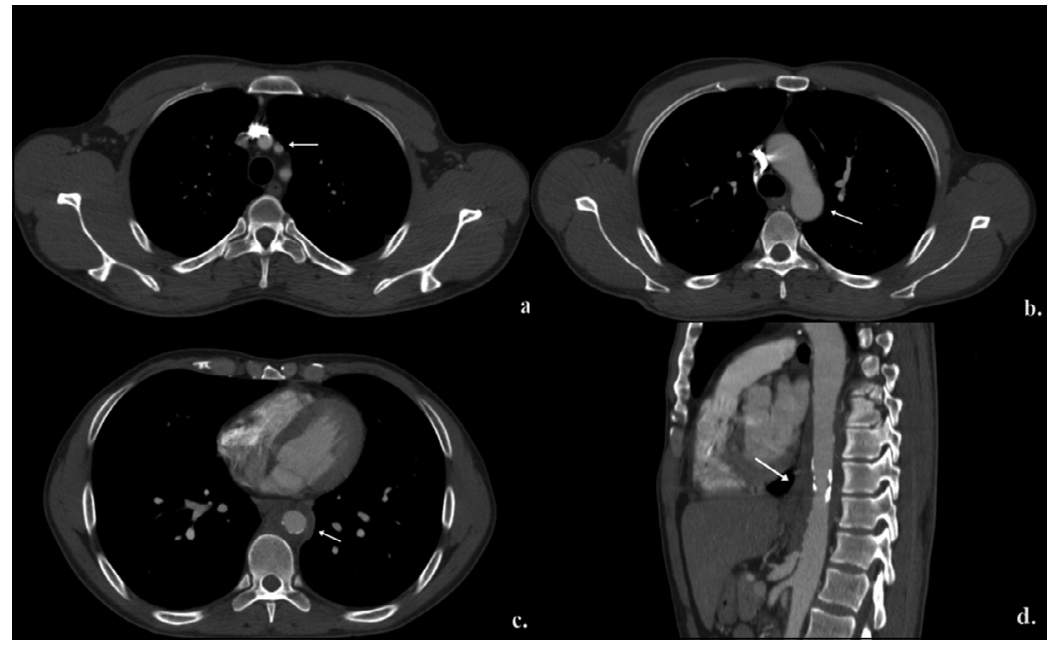

Figura 2. Cortes axiales y sagitales de TC contrastada. A nivel de los troncos supra-aórticos (a) y arco aórtico (b) no se observan evidencias de engrosamiento parietal. En la aorta descendente (c) se aprecia gran engrosamiento concéntrico y realce de las paredes extendiéndose hasta aorta abdominal proximal a nivel de la emergencia del tronco celíaco (d). 


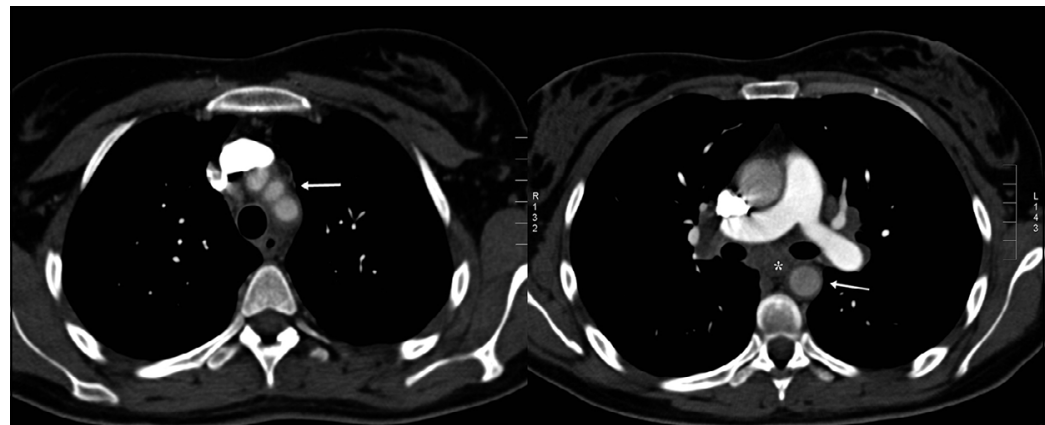

A.

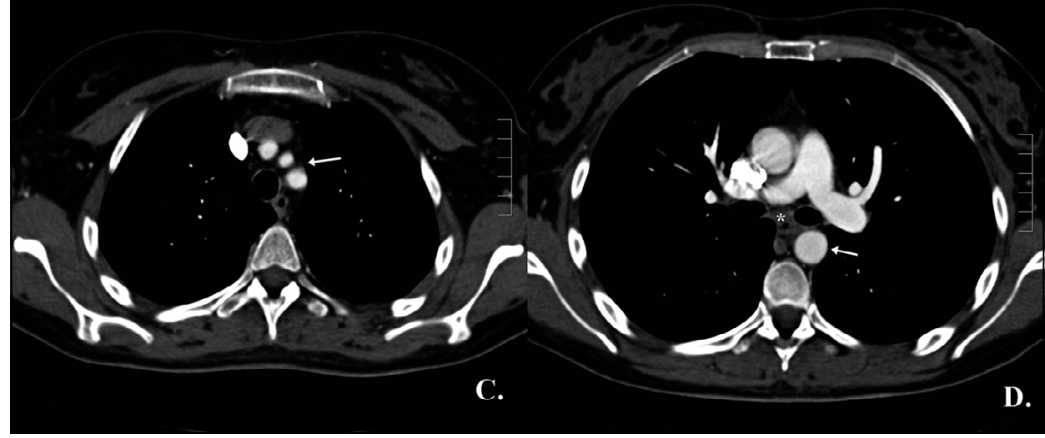

Figura 3. Cortes axiales de TC contrastada. En la fila superior, se aprecia engrosamiento concéntrico y realce de las paredes de los troncos supraaórticos (A) y aorta descendente (B). Son evidentes además numerosas adenopatías mediastínicas e hiliares bilaterales $\left(^{*}\right)$. Fila inferior, post tratamiento esteroidal. Se observa marcada regresión del compromiso de dichos troncos arteriales (C, D) y de las adenopatías mediastínicas e hiliares $\left({ }^{*}\right)$.
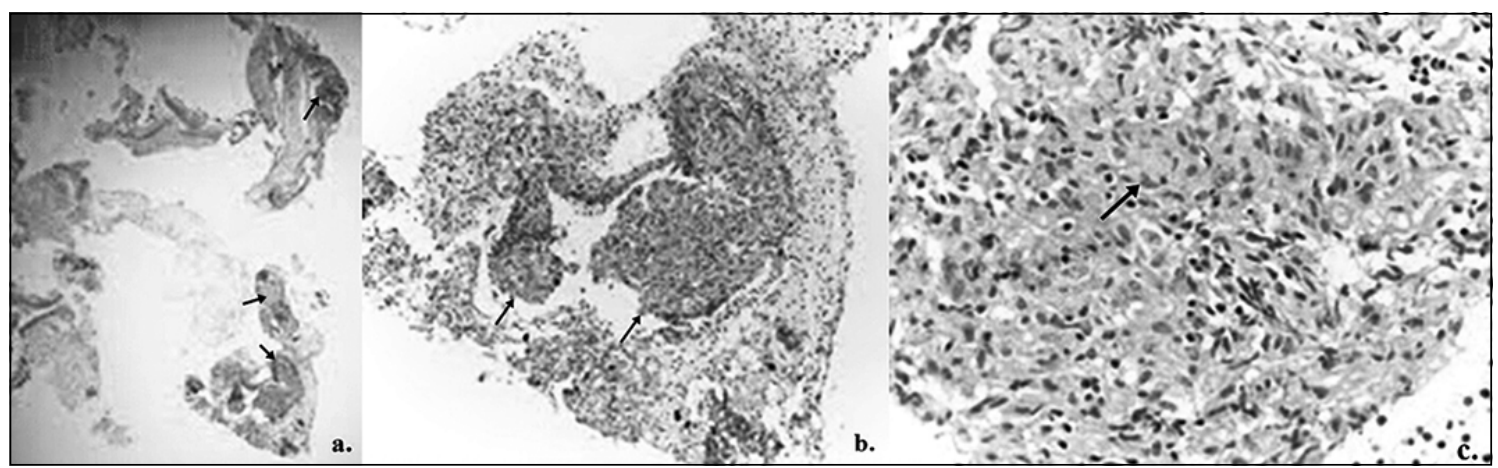

Figura 4. Biopsia trans-bronquial de adenopatía mediastínica. Tinción hematoxilina-eosina. Mucosa respiratoria con áreas nodulares perilinfáticas (flechas) 4X (a). Granulomas perilinfáticos (flechas), constituidos por macrófagos epitelioides en cúmulos, sin necrosis, sin corona linfocitaria (b). Acercamiento de granuloma 40X. Flecha indica macrófagos epitelioides (c)

\section{Discusión}

La asociación entre enfermedades reumatológicas y compromiso aórtico está bien establecida, pero su prevalencia en las distintas patologías es variable y desconocida.

En reumatología el término aortitis es asocia- do a ET y ACG, sin embargo, con una frecuencia mucho menor la inflamación de la aorta puede presentarse en otras entidades como las anteriormente mencionadas ${ }^{2}$.

El compromiso cardiovascular en el LES, además del riesgo de ateromatosis, está dado principalmente por pericarditis, endocarditis, 
miocarditis y vasculitis de pequeño vaso ${ }^{6}$. La aortitis es una manifestación muy infrecuente con aproximadamente 40 casos descritos en la literatura, la mayoría diagnosticados en relación a estudio anatomopatológico de aneurismas ${ }^{7-11}$. Se sugieren 2 mecanismos patogénicos, uno relacionado a la ateromatosis agravada por corticoides y el segundo por degeneración quística de la media secundaria a vasculitis. La mayoría de los casos se presentan con disección, aneurismas y trombos ${ }^{7}$. El caso $\mathrm{n}^{\circ} 1$ se detectó precozmente, respondió satisfactoriamente al tratamiento inmunosupresor y no presentó ninguna de estas complicaciones en el seguimiento.

En el caso no 2 el diagnóstico de GP se realizó por el compromiso ocular y pulmonar característico, los anticuerpos y la posterior biopsia. Dados estos antecedentes, los síntomas con los que debutó fueron atribuidos a la aortitis y se concluyó que esta era secundaria a la misma enfermedad, ya que no existía otra causa que la explicara. Si bien las vasculitis asociadas a ANCA afectan predominantemente vasos de pequeño calibre, existen reportes de compromiso aórtico y otros grandes vasos ${ }^{12-16}$. En aquellos con estudio histopatológico se encontró infiltrado inflamatorio mixto, especialmente alrededor de vasa vasorum y sólo algunos tenían presencia de granulomas ${ }^{16}$.

La sarcoidosis es una enfermedad granulomatosa sistémica que afecta principalmente pulmones, pero puede comprometer el sistema musculo-esquelético, piel, sistema nervioso central, entre otros. La presencia de vasculitis es una complicación inusual, pero se han descrito casos con afección de pequeños y grandes vasos. Existen unos pocos reportes de la concurrencia de sarcoidosis con aortitis ${ }^{17-20}$, algunos estiman que se trataría de ET asociado a sarcoidosis y que ambas entidades podrían tener una etiopatogenia relacionada ya que son patologías de baja frecuencia por lo que su asociación debida al azar sería rarísima. En la mayoría de los casos descritos, la sarcoidosis precede a la aortitis y que $50 \%$ cursa con uveítis ${ }^{20}$. Ninguno de estos aspectos ocurrió en nuestra paciente.

En la identificación de la causa es mandatorio la pesquisa de otros signos y síntomas de la enfermedad subyacente junto a los aspectos sociodemográficos, apoyado de análisis de laboratorio que pudiesen contribuir al diagnóstico diferencial, ya que la clínica y el estudio anatomopatológico de la aortitis no contribuyen al diagnóstico etiológico. Las imágenes podrían contribuir a diferenciar las causas infecciosas de las inflamatorias ${ }^{21}$.

El tratamiento en la mayoría de las aortitis no infecciosas es con corticoterapia con o sin otro inmunosupresor según la enfermedad subyacente. El seguimiento es clínico, con imágenes y parámetros inflamatorios. En caso de estenosis o aneurisma es fundamental la concurrencia del cirujano vascular para tratar las secuelas.

\section{Conclusiones}

Aortitis es un término inespecífico para referirse a la inflamación de la aorta. Su sintomatología es variable e inespecífica por lo que se requiere un alto índice de sospecha para diagnosticarla. La mayoría de las veces es un hallazgo fortuito. Los estudios por imágenes confirman el diagnóstico. Las etiologías son variadas entre las que existen causas infecciosas y reumatológicas. De estas últimas se han descrito en la literatura asociaciones infrecuentes con vasculitis ANCA, lupus y sarcoidosis.

\section{Referencias}

1. Schmidt J, Sunesen K, Kornum P, Duhaut P, Thomsen R. Predictors for pathologically confirmed aortitis after resection of the ascending aorta: A 12-year Danish nationwide population-based cross-sectional study. Arthritis Res Ther 2011; 13 (3): R87.

2. Gornik H, Creager M. Aortitis. Circulation 2008; 117 (23): 3039-51.

3. Steuer M, Fritsche G, Tzankov T, Sturm W, Konwalinka G, Gruber J. Large-vessel arteritis and myelodysplastic syndrome: report of two cases. Eur J Haematol 2004; 73 (2): 128-33.

4. Slobodin G, Naschitz JE, Zuckerman E, Zisman D, Rozenbaum M, Boulman N, et al. Aortic involvement in rheumatic diseases Clin Exp Rheumatol 2006; 24 (Suppl. 41): S41-7.

5. Dhaon P, Das SK, Saran RK, Parihar A. Is Aorto-arteritis a manifestation of primary antiphospholipid antibody syndrome? Lupus 2011; 20 (14): 1554-6.

6. Gómez-León A, Amezcua-Guerra LM. Cardiovascular Manifestations of systemic lupus erythematosus. Arch Cardiol Mex 2008; 78 (4): 421-30.

7. Silver AS, Shao CY, Ginzler EM. Aortitis and aortic 
Aortitis en reumatología, causas infrecuentes - P. Wurmann et al

thrombus in systemic lupus erythematosus. Lupus 2006; 15 (8): 541-3.

8. Breynaert C, Cornelis T, Stroobants S, Bogaret J. Systemic lupus erythematosus complicated with aortitis. Lupus 2008; 17: 72-4.

9. Takagi H, Mori Y, Iwata H, Kimura M, Itokazu M, Shimokawa K, et al. Nondissecting aneurysm of the thoracic aorta with arteritis in systemic lupus erythematosus. J Vasc Surg 2002; 35: 801-4.

10. Guard R, Gotis-Graham I, Edmonds J, Thomas A. Aortitis with dissection complicating systemic lupus. Pathology 1995; 27: 224-8.

11. Kurata A, Kawakami T, Sato J, Sakamoto A, Muramatsu T, Nakabayashi K. Aortic aneurysms in systemic lupus erythematosus: a meta-analysis of 35 cases in the literature and two different pathogeneses. Cardiovascular Pathology 2011; 20 (1): e1-7.

12. Oberwalder P, Tilz G, Rigler B. Spontaneous acute type A aortic dissection as a result of autoimmune aortitis without previous aortic dilatation in a 43-year-old man. J Thorac Cardiovasc Surg 2003; 125: 413.

13. Murakami A, Motomura N, Takaoka T, Kobayashi J, Maeda K, Yamamoto T, et al. Coronary-coronary bypass and pulmonary artery reconstruction related to Wegener granulomatosis in a 22-year-old woman. J Thorac Cardiovasc Surg 2003; 125: 721-3.

14. Goodfield N, Bhandari S, Plant W, Morley-Davies A, Sutherland G. Cardiac involvement in Wegener's granulomatosis. Br Heart J 1995; 73: 110-5.
15. Kim J-B, Kim W, Park M-J. Reversal of aortic root inflammation after treatment of Wegener's granulomatosis. Heart 2013; 99: 507.

16. Chirinos JA, Tamariz LJ, Lopes G, Del Carpio F, Zhang X, Milikowski C, et al. Large vessel involvement in ANCA-associated vasculitides: report of a case and review of the literature. Clin Rheumatol 2004; 23(2): 152-9.

17. Bejerano C, Blanco R, González-Vela C, Agüero R, Carril JM, González-Gay MA. Refractory polimyalgia rheumatica as presenting manifestation of large-vessel vasculitis associated to sarcoidosis. Successful response to adalimumab. Clin Exp Rheumatol 2012; 30 (1), suppl 70: S94-7.

18. Izumikawa $\mathrm{K}$, Motoi N, Takaya H, Miyamoto A, Eishi $\mathrm{Y}$, Yoshimura K, et al. A case of concurrent Sarcoidosis, Aortitis and Crohn's disease. Intern Med 2011; 50 (23): 2915-7.

19. Rafiq I, Nadig V, Freeman L. Sarcoidosis, microvascular angina and aortitis: New dimensions of the "Takayasu syndrome”. A case report. Int J Angiol 2007; 16 (3): 1134.

20. Weiler V, Redtenbacher S, Bancher C, Fischer MB, Smolen JS. Concurrence of sarcoidosis and aortitis: case report and review of the literature. Ann Rheum Dis 2000; 59: 850-3.

21. Venkata K, Restrepo C. Infectious and noninfectious Aortitis: Cross-Sectional Imaging Findings. Semin Ultrasound CT MR 2012; 33 (3): 207-21. 